\title{
Dislocation dynamics in strain relaxation in GaAsSb/GaAs heteroepitaxy
}

\author{
B. Pérez Rodríguez \\ Randall Laboratory, Applied Physics Program, University of Michigan, Ann Arbor, Michigan 48109 \\ J. Mirecki Millunchick ${ }^{\mathrm{a})}$ \\ Department of Materials Science and Engineering, University of Michigan, Ann Arbor, \\ Michigan 48109-2136
}

(Received 11 January 2006; accepted 27 May 2006; published online 18 August 2006)

\begin{abstract}
The real-time stress evolution has been investigated during molecular-beam epitaxial growth of $\mathrm{GaAs}_{1-x} \mathrm{Sb}_{x} / \mathrm{GaAs}$ metamorphic buffer. These real-time data were obtained using an in situ multibeam optical sensor measurement and has been combined with detailed analysis of data obtained from x-ray diffraction, transmission electron microscopy, and atomic force microscopy. We compare the strain relaxation of two different compositions of $\mathrm{GaAs}_{1-x} \mathrm{Sb}_{x}$, and correlated the development of dislocation structure and morphology. Several distinct stages of the strain relaxation were observed during growth, which can be separated in three main regimes: pseudomorphic growth, fast strain relaxation, and saturation. Transmission electron microscopy data show that $\mathrm{GaAs}_{0.5} \mathrm{Sb}_{0.5}$ buffer layers have a larger fraction of pure-edge dislocations that arise during the earliest stages of growth. This could have a significant influence in the fabrication of buffer layers, since pure edges are favored over the threading dislocations. The strain relaxation profile for each film was modeled using a modified model of Dodson and Tsao [Phys. Rev. B 38, 12383 (1988)] that takes into account the elastic interactions of misfit dislocations. The model results agree with the experimental data and show that interaction of misfit dislocations is responsible for the large residual stress. In addition, following the description developed by Dodson and Tsao [Phys. Rev. B 38, 12383 (1988)] for the rate of dislocation multiplication, we were able to determine the line density of threading dislocations from the experimental data. This has a potential application in the design of metamorphic buffer layers because our observations are made in real time on individual growth, without the need of external characterization to measure the dislocation density. (C) 2006 American Institute of Physics. [DOI: 10.1063/1.2245206]
\end{abstract}

\section{INTRODUCTION}

Metamorphic buffer layers have recently been proven to be a useful way to integrate high performance devices with low cost substrates. ${ }^{1}$ The success of the metamorphic approach is in the ability of the buffer layer to relax the stress due to lattice mismatch between the substrate and the epitaxial layer, without degrading the quality of the active region. Stresses that build up in the buffer layer as the film grows have been shown to result in bending of the substrate, which has been studied for $\mathrm{SiGe} / \mathrm{Si}^{2}, \mathrm{Ge} / \mathrm{Si},{ }^{3} \mathrm{Fe} / \mathrm{GaAs},{ }^{4}$ InAs $/ \mathrm{GaAs}{ }^{5}$ and InGaAs/GaAs (Ref. 6) films. There are several mechanisms by which the stress (or the strain) may be relieved. It is well known that the introduction of dislocations relaxes the strain, however, the presence of these defects in the active region degrades device properties. Therefore, one requirement for an appropriate metamorphic buffer layer is that the defect density be minimized near the active region. Also, the surface has been observed to roughen in response to the stress ${ }^{7}$ or in the presence of buried dislocations. ${ }^{8}$ The result of such roughening may be in higher carrier scattering, resulting in reduced mobility. Consequently, understanding and controlling the origin of stress is of importance to the reliable fabrication of devices upon metamorphic buffer layers. In this work, the stress evolution

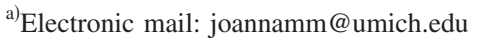

and strain relaxation for $\mathrm{GaAs}_{0.5} \mathrm{Sb}_{0.5}$ and $\mathrm{GaAs}_{0.61} \mathrm{Sb}_{0.39}$ films deposited on GaAs (001) substrates is examined and correlated to evolution of the surface morphology and the formation of misfit dislocations. The stress evolution is also compared to a modified Dodson-Tsao model that takes threading dislocation interactions into account. This model is shown to predict the linear density of dislocations within a factor of 2 or 3 .

\section{EXPERIMENT}

$\mathrm{GaAs}_{1-x} \mathrm{Sb}_{x}$ films were grown at a substrate temperature $T_{\text {sub }}=477^{\circ} \mathrm{C}$ using molecular-beam epitaxy (MBE) on 3 in. Si-doped GaAs (001) substrates with a thickness of $450 \mu \mathrm{m}$. Whole wafers were loaded on non-In-bonded sample holders to allow flexure during growth. The oxide was desorbed under an $\mathrm{As}_{2}$ overpressure at $T_{\text {sub }}=600{ }^{\circ} \mathrm{C}$, followed by the deposition of a 1470 - $\AA$-thick GaAs buffer layer at $T_{\text {sub }}$ $=573{ }^{\circ} \mathrm{C}$. Incorporation rates $R$ were calibrated using reflection high-energy electron diffraction (RHEED) prior to the growth of the film. The incorporation rates used throughout the experiment for $\mathrm{Ga}$ and As were $R_{\mathrm{Ga}}=0.96$ and $R_{\mathrm{As}}$ $=1.11$ monolayer $/ \mathrm{s}(\mathrm{ML} / \mathrm{s})$, respectively. These high rates were used to avoid any compositional variations across the film. ${ }^{9,10}$ The $\mathrm{Sb}$ incorporation rate for the $\mathrm{GaAs}_{0.5} \mathrm{Sb}_{0.5}$ film was $R_{\mathrm{Sb}}=0.61 \mathrm{ML} / \mathrm{s}$, and $R_{\mathrm{Sb}}=0.41 \mathrm{ML} / \mathrm{s}$ for the $\mathrm{GaAs}_{0.61} \mathrm{Sb}_{0.39}$ film. The stress in the $\mathrm{GaAs}_{1-x} \mathrm{Sb}_{x}$ film was 


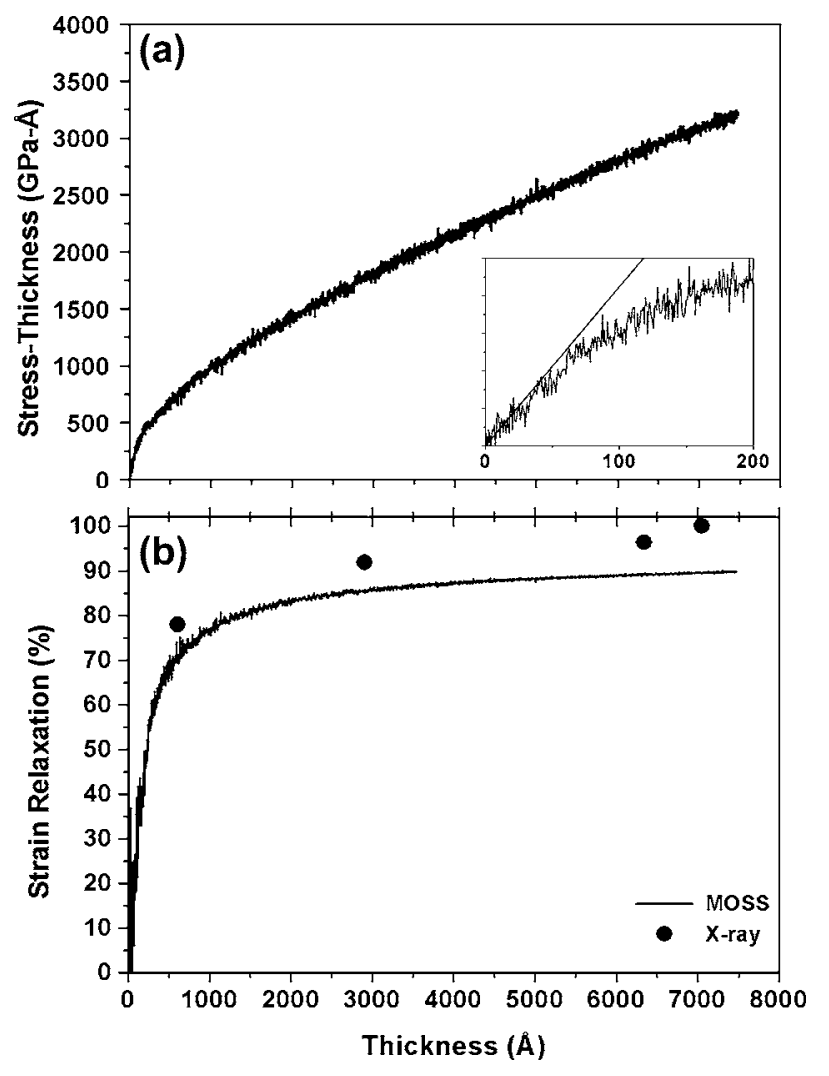

FIG. 1. (a) Stress thickness vs thickness for a $\mathrm{GaAs}_{0.5} \mathrm{Sb}_{0.5} / \mathrm{GaAs}$ film deposited at $477{ }^{\circ} \mathrm{C}$ and growth rate $r_{g}=2.99 \AA / s$. The inset shows the evolution of the stress thickness for the first $200 \AA$ of growth, and the line indicates the expected stress thickness for a coherently strained film. (b) The calculated strain relaxation vs thickness for this film. The filled circles show the strain relaxation obtained from XRD for samples grown to various thicknesses.

monitored in situ by measuring the wafer curvature using a multibeam optical sensor (MOS) technique from which the strain relaxation was calculated. ${ }^{2,6}$ The surface morphology was characterized ex situ using a tapping mode atomic force microscopy (AFM). High resolution x-ray diffraction measurements were obtained using a four crystal diffractometer. Scans about (004) and grazing exit (115) diffraction conditions were conducted for the [110] azimuth. These results were fit using a dynamical simulation in order to deduce the composition, and amount of strain relaxation in these films. The microstructure, including the dislocation density, was further characterized using transmission electron microscopy (TEM). For these analyses, cross-sectional and plan-view samples were prepared using conventional mechanical polishing and followed by argon ion milling. Cross-sectional high resolution images were obtained using JEOL 3011 transmission electron microscope operating at $300 \mathrm{kV}$. Planview images were obtained using a Philips CM12 operating at $120 \mathrm{kV}$.

\section{RESULTS}

The average stress in the film may be determined from measuring the wafer curvature during growth as described in Refs. 11 and 12. The stress-thickness evolution during the growth of the $\mathrm{GaAs}_{0.5} \mathrm{Sb}_{0.5} / \mathrm{GaAs}$ film is shown in Fig. 1(a). The inset shows the first $200 \AA$ of the growth. The straight

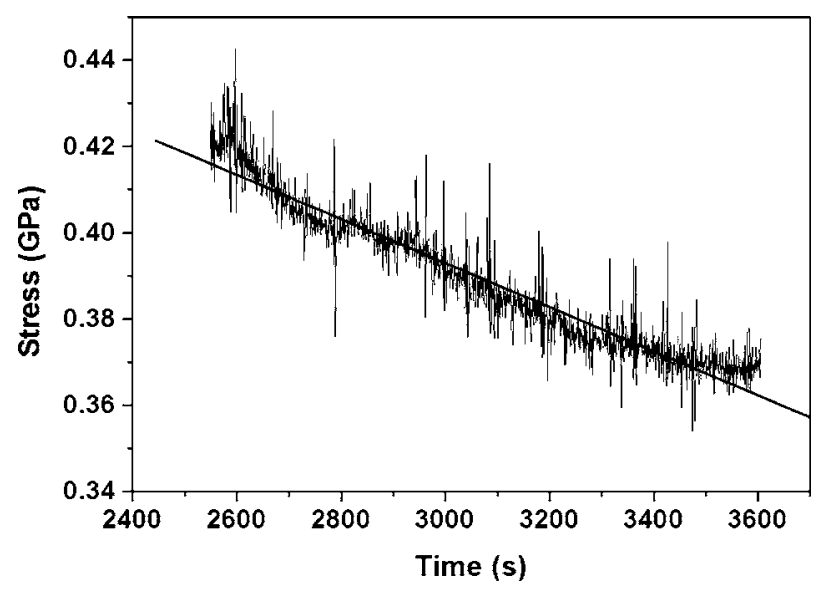

FIG. 2. Stress evolution of a $\mathrm{GaAs}_{0.5} \mathrm{Sb}_{0.5} / \mathrm{GaAs}$ film during cooling from 470 to $200^{\circ} \mathrm{C}$.

line corresponds to a coherently strained film at this composition, and it is evident that for $h_{f}<25 \AA$ no relaxation takes place. This linear increase corresponds to the pseudomorphic regime. As the film gets thicker the slope appreciably decreases due to strain relaxation. The evolution of the strain relaxation during growth is shown in Fig. 1(b), and is defined as $\gamma=\left(f_{o}-\varepsilon / f_{o}\right) \times 100$, where $f_{o}=\left(a_{s}-a_{f}\right) / a_{s}$ is the misfit strain, and $a_{s}$ and $a_{f}$ are the lattice parameters of the substrate and film, respectively. The apparently large fluctuations in the strain relaxation for $h_{f}<25 \AA$ are due to the fact the strain is calculated from the product of the stress thickness. Therefore, any small fluctuations in the stress thickness are amplified when converted to stress (or strain) by dividing by a small value of $h_{f}$. Nonetheless, we deduce that for $h_{f}$ $<25 \AA$ less than $10 \%$ of strain is relieved. Fast relaxation occurs for $25 \AA h_{f}<300 \AA$ where the film relaxes up to $60 \%$ of the strain with a rate of $0.2 \% / \AA$. Beyond $300 \AA$, the rate of strain relief continues to decay for the duration of the growth with a rate of $0.0013 \% / \AA$. Figure 1(b) shows that at $h_{f}=7050 \AA$, roughly $82 \%$ of the strain has relaxed.

In order to verify the composition $x$ and percent strain relaxation $\gamma$, symmetric and grazing exit scans were obtained from rocking curves in samples grown to thicknesses of $h_{f}$ $=610,2900,6340$, and $7100 \AA$. The results of these measurements are shown as points in Fig. 1(b). Although the $x$ obtained from x-ray diffraction (XRD) is in good agreement with the MOS data, $\gamma$ is higher in some cases by $13 \%$. This is in contrast to reports in other systems such as $\operatorname{In}_{0.2} \mathrm{Ga}_{0.8} \mathrm{As}$ (Refs. 12 and 13) and $\mathrm{In}_{0.12} \mathrm{Ga}_{0.88} \mathrm{As}_{0.99} \mathrm{~N}_{0.01},{ }_{14}$ where $\gamma$ obtained by XRD and MOSS is within 3\%. This suggests that additional relaxation may occur during cooling. Figure 2 shows the stress evolution versus time during cooling from $T=470{ }^{\circ} \mathrm{C}$ to $T=200{ }^{\circ} \mathrm{C}$, and shows that the film stress becomes less compressive. A linear fit of this portion of the data shows that the thermal stress induced over this period is $\sigma_{\mathrm{th}}=0.05 \mathrm{GPa}$. The thermally induced strain may be obtained from the difference of substrate and film thermal strain, which is given by: ${ }^{15}$

$$
\varepsilon_{\mathrm{th}}=\left(\alpha_{S}-\alpha_{F}\right) \delta T,
$$

where $\alpha_{S}=6.93 \times 10^{-6} \mathrm{~K}^{-1}$, the coefficient of thermal expansion of the GaAs substrate, $\alpha_{F}=7.90 \times 10^{-6} \mathrm{~K}^{-1}$, the coeffi- 


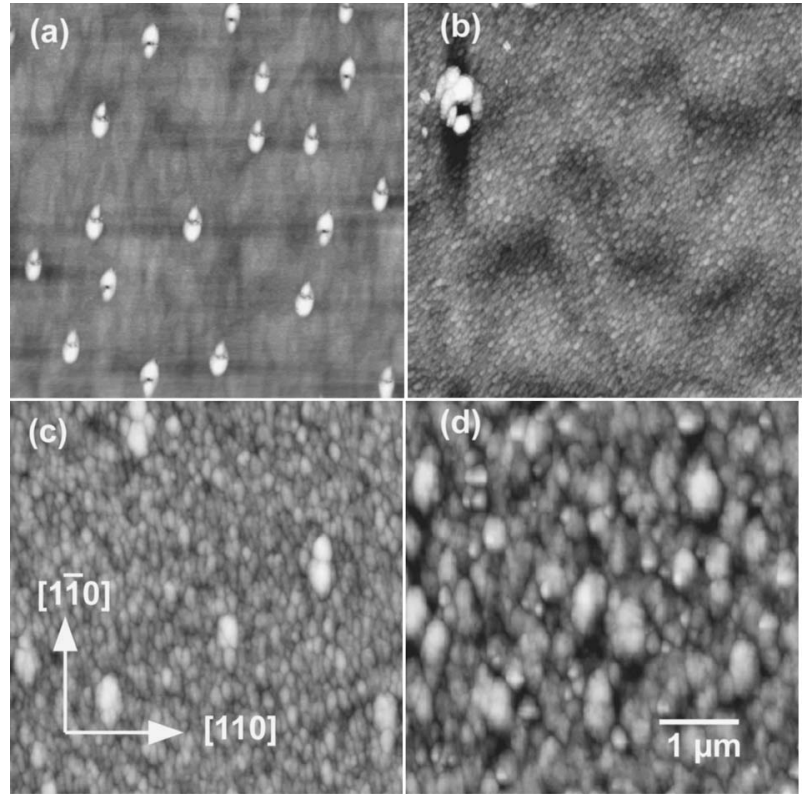

FIG. 3. $5 \times 5 \mu \mathrm{m}^{2} \mathrm{AFM}$ images for $h_{f}=$ (a) $65 \AA$, (b) $173 \AA$, (c) $2906 \AA$, and (d) $7050 \AA \mathrm{GaAs}_{0.5} \mathrm{Sb}_{0.5}$ samples.

cient of thermal expansion of the $\mathrm{GaAs}_{0.5} \mathrm{Sb}_{0.5}$ interpolated from the values of pure GaAs and $\mathrm{GaSb},{ }^{16,17}$ and $\delta T$ $=-270 \mathrm{~K}$, the drop in the temperature during cooling. Since the substrate is much thicker than the film, we assume that the difference in temperature between substrate and film is negligible. The induced strain thus calculated is $\varepsilon_{\mathrm{th}}=0.26$ $\times 10^{-3}$, which corresponds to an induced tensile stress $\sigma_{\text {th }}$ $=0.03 \mathrm{GPa}$, and is in reasonable agreement with the measured stress. The remainder of the stress in the film is likely generated during cooling from $T=200{ }^{\circ} \mathrm{C}$ to room temperature. The predicted induced stress for $\mathrm{In}_{0.2} \mathrm{Ga}_{0.8} \mathrm{As}$ and $\mathrm{In}_{0.12} \mathrm{Ga}_{0.88} \mathrm{As}_{0.99} \mathrm{~N}_{0.01}$ at this cooling rate is $\sigma_{\text {th }}=-0.01 \mathrm{GPa}$ and $\sigma_{\text {th }}=-0.005 \mathrm{GPa}$, respectively. In both cases, the magnitude of the thermal stress is quite small, and the sign of the stress is negative. Therefore, further relaxation in these films is not expected upon cooling. Thus, the MOS and XRD data for those materials systems should be nominally the same, in agreement with the published experimental data. ${ }^{12-14}$

The morphology at various points of the strain evolution was also examined. Figure 3 shows AFM micrographs of films at various points along the strain relaxation curve. These data show that the surface topography develops quite differently within each of these three strain relaxation regimes. The boundary between the pseudomorphic and the fast relaxation regime [Fig. 3(a)] is characterized by the nucleation of large island of $0.5-1.0 \mu \mathrm{m}$ long by $0.2 \mu \mathrm{m}$ wide elongated along [1 $1 \overline{1} 0]$. These large islands are not likely oval defects because the appearance of these features depends on group V flux and their density decreases as the film thickness increases, while the density of oval defects has been observed to be independent of the III/V flux ratio and to increase with film thickness. ${ }^{18}$ Furthermore, oval defects vary in size between $3-15 \mu \mathrm{m},{ }^{19}$ contrary to what we observe. During the fast relaxation regime, smaller isotropic island of approximately $82 \mathrm{~nm}$ in diameter nucleates, as seen in Fig. 3(b). Figure 3(c) shows a decrease in the smaller

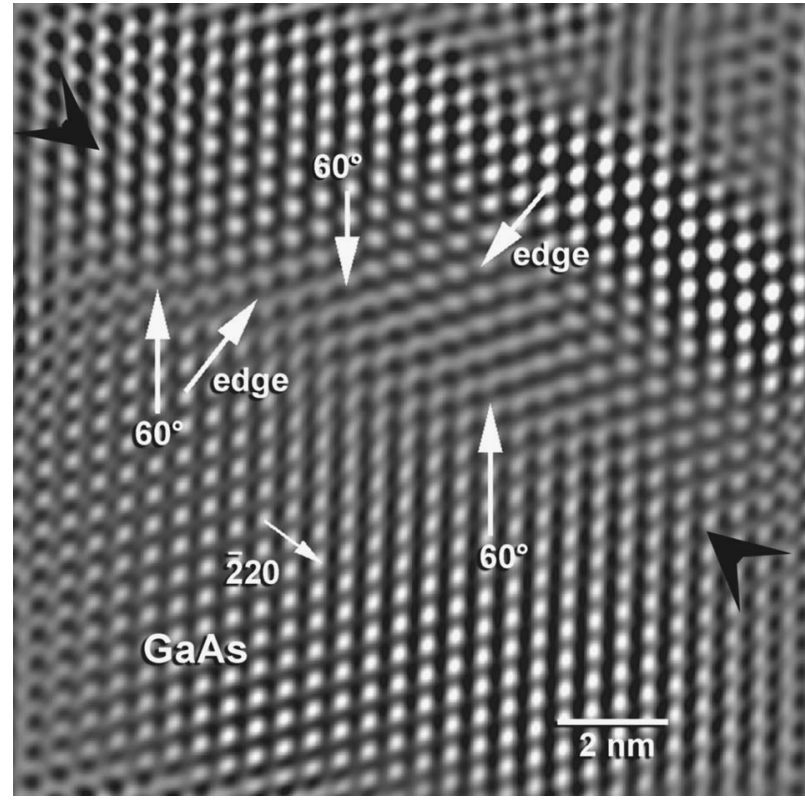

FIG. 4. Fourier-filtered (110) cross-sectional HRTEM image of 65 - $\AA$-thick $\mathrm{GaAs}_{0.5} \mathrm{Sb}_{0.5}$ film. The interface is marked by the black arrows, and $60^{\circ}$ and pure-edge misfit dislocations are indicated by white arrows.

island density due to coalescence as the strain relaxation reaches saturation. The surface remains rough at large thickness [Fig. 3(d)] likely due to the enduring coherent strain.

We have examined the dislocation structure at the epilayer/substrate interface using (110) cross-sectional high resolution TEM (HRTEM). Figure 4 shows a Fourier-filtered (110) cross-sectional HRTEM image for $\mathrm{GaAs}_{0.5} \mathrm{Sb}_{0.5}$ film at a thickness $h_{f}=65 \AA$. Dislocations in this image are identified by arrows along the $\{111\}$ and $\{110\}$ planes. Two pureedge dislocations, which are distinguish by an extra $\{110\}$ plane, and three $60^{\circ}$ dislocations, which have one extra $\{111\}$ half plane, are apparent in this image. The dislocation density was determined using this Fourier-filtered technique for several sections taken from the film near the beginning of the fast relaxation regime $\left(h_{f}=65 \AA\right)$, and another at the end $\left(h_{f}=342 \AA\right)$. The line density of each type of dislocation and average spacing $p$ are summarized in Table I. For the $h_{f}$ $=65 \AA$ film, the linear density of $60^{\circ}$ dislocations is higher than the density of pure-edge dislocations by nearly a factor of 2 . For the $h_{f}=342 \AA$ thick film, the density of $60^{\circ}$ dislocations doubles, while the density of pure-edge dislocations quadruples, indicating that pure-edge dislocations have a higher nucleation rate in the fast relaxation regime. This re-

TABLE I. Summary of the density of different kinds of dislocations in $\mathrm{GaAs}_{1-x} \mathrm{Sb}_{x}$ observed by HRTEM.

\begin{tabular}{ccccc}
\hline \hline & & \multicolumn{3}{c}{$\begin{array}{c}\text { Interface dislocation line density } \\
\left(\times 10^{6} \mathrm{~cm}^{-1}\right)\end{array}$} \\
\cline { 3 - 4 } & & \multicolumn{3}{c}{$\begin{array}{c}\text { Pure } \\
\text { edge }\end{array}$} \\
$\begin{array}{ccccc}\text { \% of Sb } \\
\text { content }\end{array}$ & $\begin{array}{c}\text { Thickness } \\
(\AA)\end{array}$ & $60^{\circ}$ & 0.7 & $83 \pm 13$ \\
\hline 50 & 65 & 1.3 & 2.6 & $70 \pm 5$ \\
50 & 342 & 2.7 & 1.2 & $100 \pm 14$ \\
39 & 1818 & 1.7 & & \\
\hline
\end{tabular}



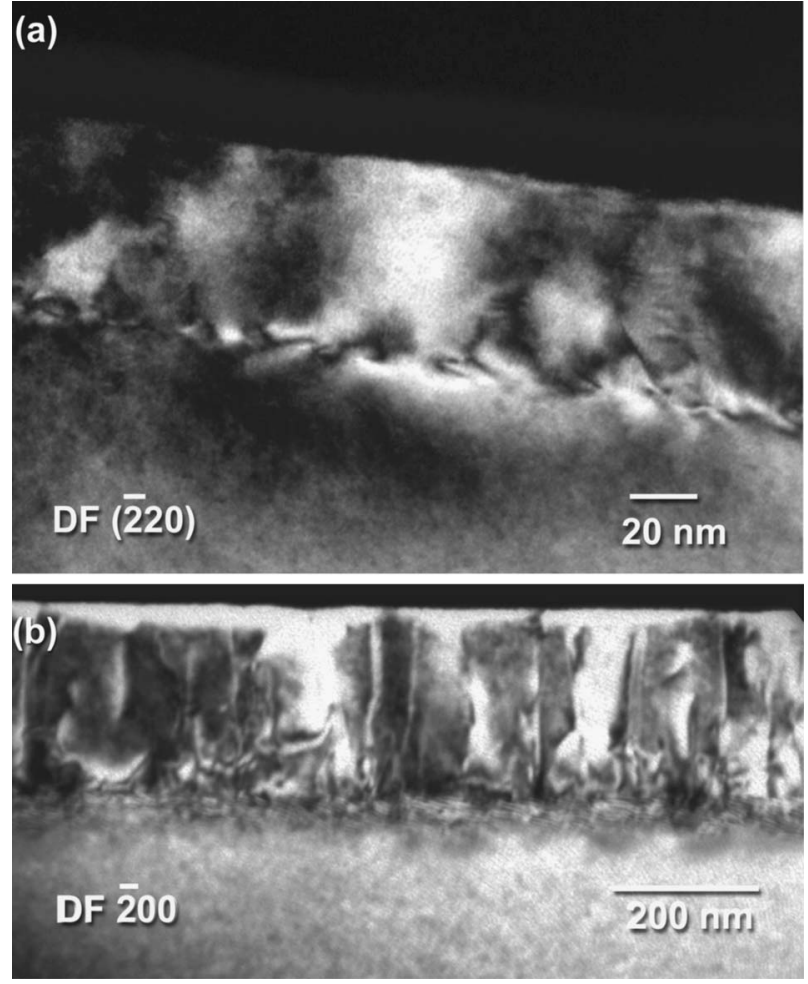

FIG. 5. Cross-sectional dark filed TEM images for (a) $608 \AA$, and (b) 2906- $\AA$-thick $\mathrm{GaAs}_{0.5} \mathrm{Sb}_{0.5}$ samples.

gime is also marked by the formation and growth of many small islands as seen in Fig. 3(b), which likely inject edge dislocations at their edge. ${ }^{20,21}$

We also examined the evolution of threading dislocations beyond the fast relaxation regime. Figure 5(a) shows a dark-field TEM image of a film at $h_{f}=608 \AA$, which is near the transition from the fast to the saturation regime. Apparent in the image are misfit dislocations at the interface and a small number of threading dislocations in the bulk of the film. As the film gets thicker, the threading dislocation density increases drastically, as seen in Fig. 5(b). Also apparent in this image are dislocations threads in a direction parallel to the growth direction, which are likely blocked threading segments due to the repulsive interaction of the stress field of a threading dislocation with an existing group of pure-edge dislocations. ${ }^{22}$ Although a few threading dislocations are present during the fast relaxation regime, it is interesting to note that they do not multiply until island coalescence begins, as indicated in the AFM images in Figs. 3(b) and 3(c). These results suggest that controlling the growth parameters such that the nucleation and growth of islands is minimized, the formation of defects may be reduced.

We also measured the average dislocation line density for the threading dislocations for various thicknesses. The average dislocation line density was found to be 1.1 $\times 10^{5} \mathrm{~cm}^{-1}$ at $h_{f}=2908 \AA$ over a range of approximately $6.8 \mu \mathrm{m}$. For thicknesses beyond $h_{f}=2908 \AA$, TEM images do not reveal a significant increase in the threading dislocation line density. The area dislocation density using planview TEM are $7.9 \times 10^{9} \mathrm{~cm}^{-2}$ for $h_{f}=2908 \AA$ and 1 $\times 10^{10} \mathrm{~cm}^{-2}$ for $h_{f}=7100 \AA$. This suggests that dislocation interactions dominate once this high linear density is

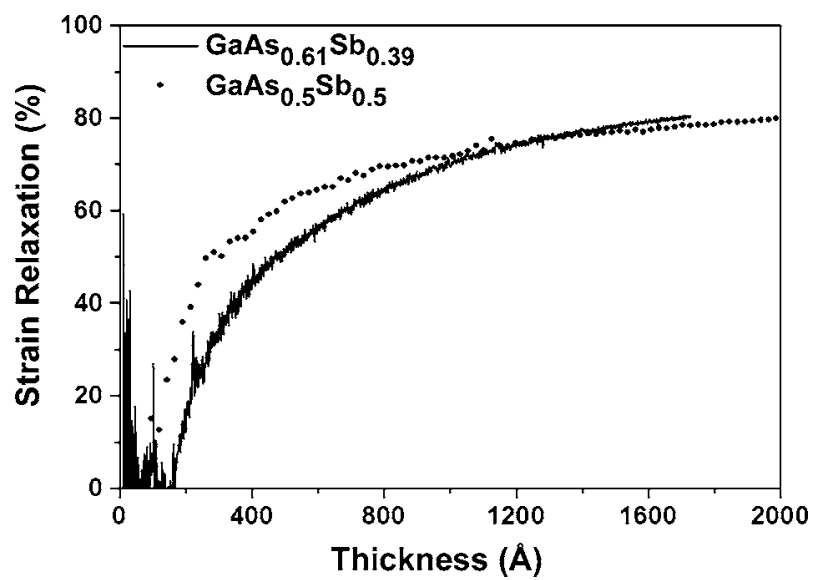

FIG. 6. Comparison between the strain relaxation for $\mathrm{GaAs}_{0.61} \mathrm{Sb}_{0.39}$ and $\mathrm{GaAs}_{0.5} \mathrm{Sb}_{0.5}$ films, deposited at $477{ }^{\circ} \mathrm{C}$ and with $\mathrm{Sb}$ rate of 0.41 and $0.61 \mathrm{ML} / \mathrm{s}$, respectively.

achieved. Several mechanisms are possible that can prevent complete strain relaxation, such as annihilation between edge dislocations or repulsive interactions between dislocations. ${ }^{23}$

The stress evolution for lower $\mathrm{Sb}$ composition layers was also examined. We grew $\mathrm{GaAs}_{0.61} \mathrm{Sb}_{0.39} / \mathrm{GaAs}$ layers, which have a lattice mismatch of $f_{o}=3.1 \%$, to a thickness of $h_{f}=1818 \AA$. The lower $\mathrm{Sb}$ composition was obtained by changing the $\mathrm{Sb}$ valve position until an incorporation rate of $R_{\mathrm{Sb}}=0.41 \mathrm{ML} / \mathrm{s}$ was reached, while keeping the $\mathrm{Ga}$, As, and growth temperature at the same values as for the $\mathrm{GaAs}_{0.5} \mathrm{Sb}_{0.5}$ layer. Figure 6 shows a comparison of the strain relaxation for the $\mathrm{GaAs}_{0.61} \mathrm{Sb}_{0.39}$ (solid line) and $\mathrm{GaAs}_{0.5} \mathrm{Sb}_{0.5}$ (dotted line) films. As for the more highly mismatched film, the strain relaxation evolution can be divided into three regimes; pseudomorphic, fast relaxation, and saturation. For the $\mathrm{GaAs}_{0.61} \mathrm{Sb}_{0.39}$ film, the pseudomorphic regime is larger, $h_{f}<160 \AA$, as expected due to its lower lattice mismatch. Fast relaxation occurs for $160 \AA<h_{f}<413 \AA$, where the film relaxes up to $47 \%$ of the strain with a rate of $0.2 \% / \AA$. Beyond $413 \AA$, the rate of strain relief continues to decay for the duration of the growth to approximately $0.015 \% / \AA$. At $h_{f}=1725 \AA$, roughly $82 \%$ of the strain has relaxed. Interestingly, the lower mismatch film relaxes at a faster rate in the saturation regime, resulting in a lower residual strain than its higher misfit counterpart.

The morphology at various thicknesses was examined for the $\mathrm{GaAs}_{0.61} \mathrm{Sb}_{0.39}$ films. Figure 7(a) shows the nucleation of large islands upon a smooth wetting layer, similar to the morphology observed for $\mathrm{GaAs}_{0.5} \mathrm{Sb}_{0.5}$ in the pseudomorphic regime [Fig. 3(a)], except that the density of these islands is lower. As the thickness of the film increases, the islands form pits at their center, and small islands with an average diameter of $62 \mathrm{~nm}$ form, as seen in Fig. 7(b). During the saturation regime, the smaller islands coalesce forming ridges along [1ㅣㅣ, and the large islands disappear [Fig. 7(c)]. Cross-sectional TEM images over a range of $2.7 \mu \mathrm{m}$ indicate a lower density of dislocation walls, thus a lower density of pure-edge dislocations, as seen in Fig. 8. From these images, the line density of threading dislocations was found to be $2.2 \times 10^{5} \mathrm{~cm}^{-1}$. An areal threading dislocation density of $5.39 \times 10^{9} \mathrm{~cm}^{-2}$ was measured using plan-view TEM for a 

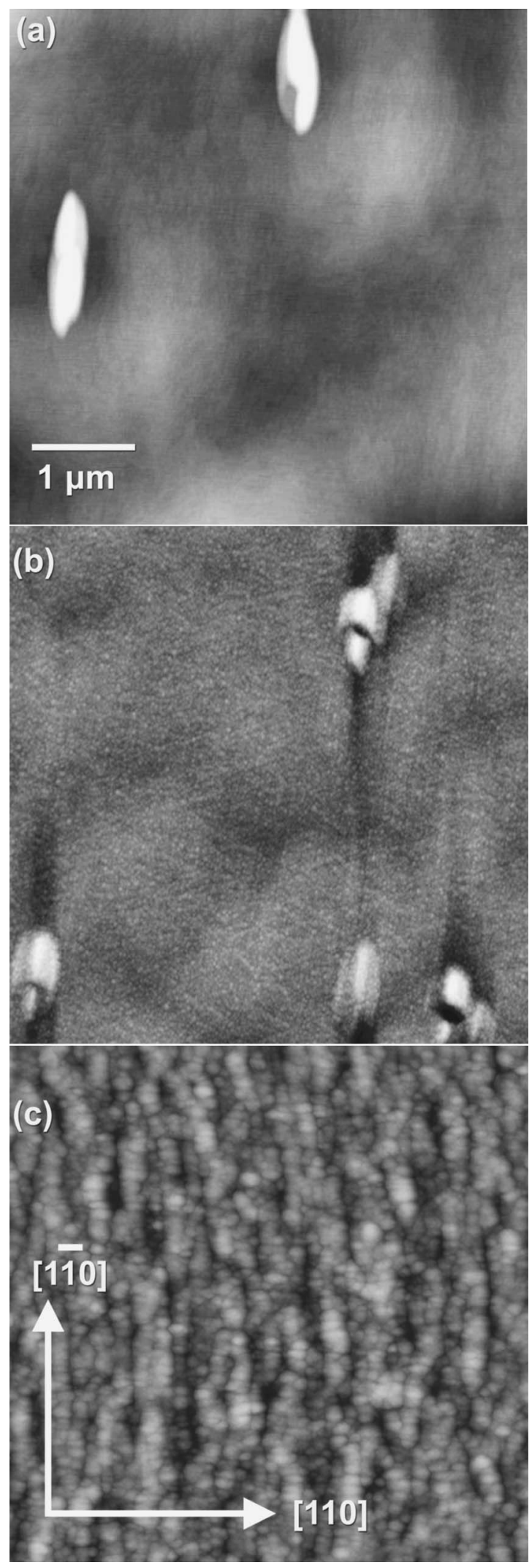

FIG. 7. $5 \times 5 \mu \mathrm{m}^{2}$ AFM images for $h_{f}=$ (a) $64 \AA$, (b) $333 \AA$, and (c) $1814 \AA \mathrm{GaAs}_{0.61} \mathrm{Sb}_{0.39}$ samples.

$\mathrm{GaAs}_{0.61} \mathrm{Sb}_{0.39}$ film at $h_{f}=1818 \AA$. Table I shows a smaller density of pure-edge dislocations relative to the obtained for $\mathrm{GaAs}_{0.5} \mathrm{Sb}_{0.5}$ films, while the number of threading dislocations are on the same order of magnitude.

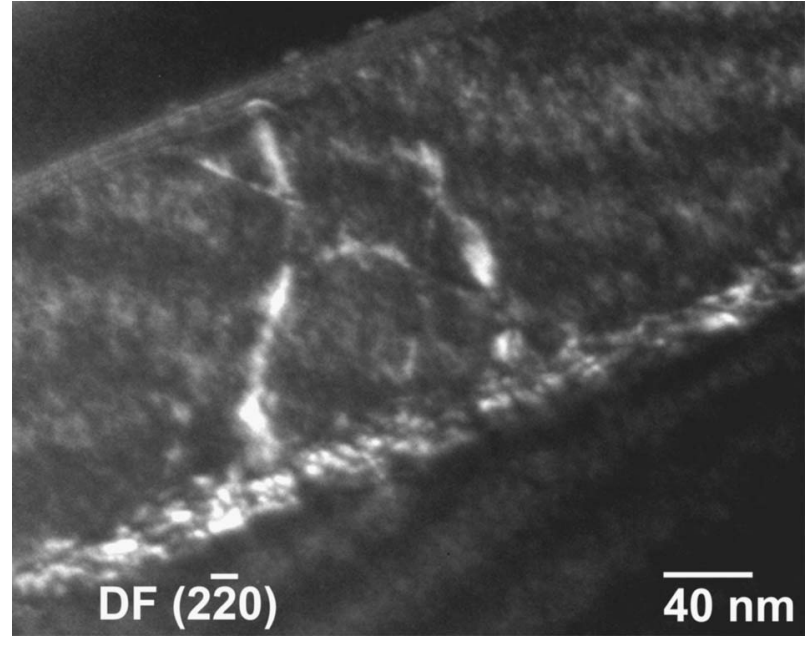

FIG. 8. Dark-field, $g=(\overline{2} 20)$, cross-sectional TEM of $\mathrm{GaAs}_{0.61} \mathrm{Sb}_{0.39}$ at $h_{f}$ $=1818 \AA$.

\section{DISCUSSION}

The dynamics of dislocation motion are crucial in the strain relaxation in thin films. It has been postulated that dislocations move through the film by a combined effect of glide and climb. ${ }^{23-26}$ Dodson and Tsao have shown that the strain relaxation rate may be expressed as a single nonlinear differential equation in thickness $h$,

$$
\frac{d \gamma}{d h}=\frac{C M^{2}}{r_{g} \sigma_{o}^{2}}\left[f_{o}-\gamma(h)-r(h)\right]^{2}\left[\gamma(h)+\gamma_{o}\right],
$$

where $r_{g}$ is the growth rate, $f_{o}$ is the lattice mismatch, and $\gamma_{o}$ is a fitting parameter that represents the initial strain relief due to preexisting misfit dislocations. $C M^{2} / \sigma_{o}^{2}$ is a fitting parameter where $C \propto e^{-U / k T}$. The term $r(h)$ is the equilibrium residual strain arising due to the dislocations. Because pureedge dislocations are sessile, we only consider the contribution of the mobile threading dislocations. Using the classic treatment from Hirth and Lothe, the equilibrium residual strain may be calculated, ${ }^{27}$

$$
r(h)=\frac{b_{60}\left(1-\nu \cos ^{2} \theta\right)}{8 \pi(1+\nu) R_{h, p} \cos \lambda} \ln \left(\frac{\alpha_{c} R_{h, p}}{b}\right),
$$

where $b_{60}=a / 2 \sqrt{2}$ is the Burger's vectors of threading dislocations, $\nu$ is the Poisson ratio, $\alpha_{c}$ is the core cutoff parameter, and $\theta$ is the angle between the dislocation line and the Burger's vector. $R_{h, p}$ is called the complex modulus of dislocation semispacing, which is the approximate solution for a stressfree boundary surface associated with two free cylinder surfaces. When we consider only the image dislocation outside the film, the self-stress of a misfit dislocation is $R_{h, p} \sim h$, where $h$ is the film thickness. However, as Fischer et al. point out, ${ }^{28}$ dislocations in the film not only interact with their image, but with the real stress fields of misfit dislocation in the interface. For an ideal case, the complex dislocation semispacing term for the self-stress of a real symmetrical interfacial misfit dislocation is approximately one half of the dislocation spacing. ${ }^{27}$ However, due to the uneven distribution of the stress field and the asymmetry of the dislocation spacing in the interface, the complex dislocation semis- 

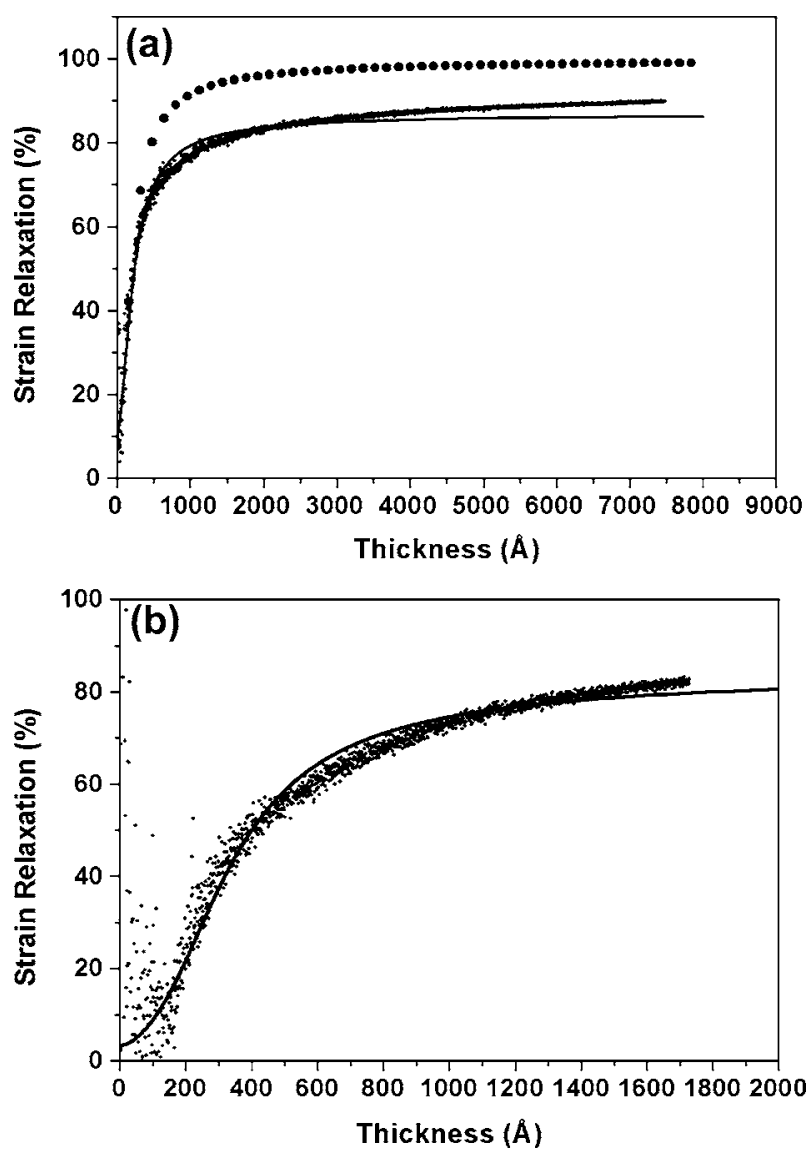

FIG. 9. Comparison of the strain relaxation model with (straight line) and without (dashed line) the dislocation interaction term, to the experimental data (points) for (a) $\mathrm{GaAs}_{0.5} \mathrm{Sb}_{0.5}$ and (b) $\mathrm{GaAs}_{0.61} \mathrm{Sb}_{0.39}$ films.

pacing term for a real misfit dislocation may be approximated $R_{h, p} \sim p$, where $p$ is the misfit dislocation spacing. Taking the interactions between the image dislocations and the dislocations in the interface, ${ }^{28}$ the complex modulus becomes

$$
R_{h, p}=\left(\frac{1}{h^{2}}+\frac{1}{p^{2}}\right)^{1 / 2} .
$$

It should be noted that in this form of the equation $R_{h, p} \sim h$ for small thicknesses, and $R_{h, p} \sim p$ for large thicknesses. The $p$ may be measured experimentally by TEM or XRD for each sample. However, we have found that these values compare well to the predicted value of $p$ based solely upon lattice mismatch considerations as described by the expression

$$
p=\frac{a_{\text {Film }} a_{\text {Sub }}}{2\left|a_{\text {Film }}-a_{\text {Sub }}\right|} .
$$

The results of this model with and without the dislocation interaction are plotted in Fig. 9(a) and compared to the strain relaxation data for $\mathrm{GaAs}_{0.5} \mathrm{Sb}_{0.5}$. As shown in previous reports, ${ }^{11,29}$ the model does not agree well with actual strain relaxation data beyond the fast relaxation regime when $R_{h, p}$ is taken to be the thickness of the film. However, when $R_{h, p}$ takes dislocation interactions within the interface into account, the model fits the data well into the saturation regime. The fidelity of the fit degrades by $4 \%-6 \%$, however, at the largest thicknesses studied here.

It is now possible to obtain linear dislocation densities from these fits. The line density of misfit dislocation is related to the strain relief $\gamma$ by

$$
\gamma=b \rho \cos (\lambda) .
$$

Using the phenomenological description of the rate of dislocation multiplication described by Dodson and Tsao, ${ }^{26}$ the linear dislocation density is given by

$$
\rho=\frac{d \gamma}{d t} \frac{1}{b \cos (\lambda)\left(C M^{2} / \sigma_{o}^{2}\right) \varepsilon_{\mathrm{eff}}^{2}},
$$

where the effective stress $\varepsilon_{\text {eff }}$ and strain relief rate $d \gamma / d t$ are the in situ strain measurements and $b \cos (\lambda)=b_{60}$. Table II compares the dislocation line densities obtained experimentally from TEM images to those predicted by the model. In the saturation regime of $\mathrm{GaAs}_{0.5} \mathrm{Sb}_{0.5}$ film, the estimated dislocation line density $\rho^{\text {model }}=7.4 \times 10^{4} \mathrm{~cm}^{-1}$, which is in reasonable agreement with the experimental values $\rho^{\exp }=1.1$ $\times 10^{5} \mathrm{~cm}^{-1}$. Similarly, for $\mathrm{GaAs}_{0.61} \mathrm{Sb}_{0.39}$, the dislocation line density was estimated to be $\rho^{\text {model }}=1.9 \times 10^{5} \mathrm{~cm}^{-1}$ which is also in very good agreement with the experimental values of $\rho^{\exp }=2.2 \times 10^{5} \mathrm{~cm}^{-1}$. In order to verify that the model is applicable to other material systems, we also fit the experimental data for the strain relaxation for $\operatorname{In}_{0.2} \mathrm{Ga}_{0.8} \mathrm{As}$ grown on GaAs. In all the three cases, the experimentally observed values are about a factor of 2 higher than the predicted values. This is consistent with the observation that the models consistently underestimate the strain relaxation in these films. Another source of the discrepancy may be in the experimental measurement technique. The strain relaxation data from which the dislocation density is predicted are taken over a large portion of the sample (typically a few square centimeters), whereas the dislocation density is measured from several TEM samples and represent a much smaller area of the sample. Nonetheless, this model shows that the linear dislocation density can be estimated in real time, without the necessity of etching or sample preparation for TEM imaging.

\section{CONCLUSIONS}

The real-time strain relaxation was investigated in $\mathrm{GaAs}_{1-x} \mathrm{Sb}_{x}$ films with nominal Sb compositions of $x=0.5$

TABLE II. Comparison of the threading dislocation line density obtained experimentally and from the model.

\begin{tabular}{cccccc}
\hline \hline Film & $d \gamma / d t\left(\mathrm{~s}^{-1}\right)$ & $\varepsilon_{\text {eff }}$ & $\mathrm{CM}^{2} / \sigma_{o}^{2}\left(\mathrm{~s}^{-1}\right)$ & $\rho^{\text {model }}\left(\mathrm{cm}^{-1}\right)$ & $\rho^{\text {expt }}\left(\mathrm{cm}^{-1}\right)$ \\
\hline $\mathrm{GaAs}_{0.5} \mathrm{Sb}_{0.5}$ & $7.61 \times 10^{-7}$ & $4.06 \times 10^{-3}$ & 30 & $7.4 \times 10^{4}$ & $1.1 \pm 0.3 \times 10^{5}$ \\
$\mathrm{GaAs}_{0.61} \mathrm{Sb}_{0.39}$ & $7.54 \times 10^{-6}$ & $5.53 \times 10^{-3}$ & 62 & $1.9 \times 10^{5}$ & $2.2 \pm 0.6 \times 10^{5}$ \\
$\mathrm{In}_{0.2} \mathrm{Ga}_{0.8} \mathrm{As}$ & $3.98 \times 10^{-7}$ & $2.54 \times 10^{-3}$ & 110 & $2.8 \times 10^{4}$ & $4.9 \pm 2.5 \times 10^{4}$ \\
\hline \hline
\end{tabular}


and $x=0.39$. The strain relaxation goes through several regimes, which are correlated to the surface morphology and the nucleation of dislocations. The fast relaxation regime is characterized by the nucleation of small islands and an abrupt increase in the number of edge dislocations, while the saturation regime is distinguished by coalesce of small islands. The data were compared to a dislocation kinetic model developed by Dodson and Tsao with the addition of a dislocation interaction term, which results in a reasonable fit to the data. Using this model, estimated line dislocation densities are in good agreement with the values obtained experimentally. These results indicate that it is possible to estimate the dislocation density in real time using this wafer curvature method.

\section{ACKNOWLEDGMENT}

The work reported here is supported by a grant from the National Science Foundation under Award No. DMR0092602.

${ }^{1}$ Y. Cordier, J. M. Chauveau, D. Ferre, and J. Dipersio, J. Vac. Sci. Technol. B 18, 2513 (2000).

${ }^{2}$ J. A. Floro, E. Chason, and S. R. Lee, Mater. Res. Soc. Symp. Proc. 406, 491 (1996).

${ }^{3}$ G. Wedler, J. Walz, T. Hesjedal, E. Chilla, and R. Koch, Phys. Rev. Lett. 80, 2382 (1998).

${ }^{4}$ G. Wedler, B. Wassermann, R. Nötzel, and R. Koch, Appl. Phys. Lett. 78, 1270 (2001).

${ }^{5}$ J. M. Garcia, J. P. Silveria, and F. Briones, Appl. Phys. Lett. 77, 409 (2000).

${ }^{6}$ E. Chason, Y. Yin, K. Tetz, R. Beresford, L. B. Freund, M. Ujue Gonzalez, and J. A. Floro, Mater. Res. Soc. Symp. Proc. 583, 167 (2000).

${ }^{7}$ A. G. Cullis, A. J. Pidduck, and M. T. Emeny, Phys. Rev. Lett. 75,
2368 (1995).

${ }^{8}$ A. M. Andrews, J. S. Speck, A. E. Romanov, M. Bobeth, and W. Pompe, J. Appl. Phys. 91, 1933 (2002).

${ }^{9}$ R. Kaspi, W. T. Cooley, and K. R. Evans, J. Cryst. Growth 173, 5 (1997).

${ }^{10}$ A. Bosacchi et al., J. Cryst. Growth 201/201, 858 (1999).

${ }^{11}$ R. Beresford, J. Yin, K. Tetz, and E. Chason, J. Vac. Sci. Technol. B 18, 1431 (2000).

${ }^{12}$ B. Pérez Rodríguez and J. Mirecki Millunchick, J. Cryst. Growth 264, 64 (2004).

${ }^{13}$ R. Beresford, K. Tetz, E. Chason, and M. U. González, J. Vac. Sci. Technol. B 19, 1572 (2001).

${ }^{14}$ M. Adamcyk, J. H. Schmid, T. Tiedje, A. Koveshnikov, A. Chahboun, V. Fink, and K. L. Kavanagh, Appl. Phys. Lett. 80, 4357 (2002).

${ }^{15}$ J.-H. Zhao, T. Ryan, P. S. Ho, A. J. McKerrow, and W.-Y. Shih, J. Appl. Phys. 85, 6421 (1999).

${ }^{16}$ V. Kumar and B. S. R. Sastry, Cryst. Res. Technol. 36, 565 (2001).

${ }^{17}$ A. Zubrilov, in Properties of Advanced Semiconductor Materials GaN, $A l N, I n N, B N, S i C, S i G e$, edited by M. E. Levinshtein, S. L. Rumyantsev, and M. S. Shur (Wiley, New York, 2001), pp. 49-66.

${ }^{18}$ N. J. Kadhim and D. Mukherjee, J. Mater. Sci. Lett. 17, 595 (1998).

${ }^{19}$ N. J. Kadhim and D. Mukherjee, Vacuum 55, 249 (1999).

${ }^{20}$ A. Trampert, E. Tournie, and K. H. Ploog, Appl. Phys. Lett. 66, 2265 (1995).

${ }^{21}$ W. Qian, M. Skowronski, R. Kaspi, M. De Graef, and V. P. Dravid, J. Appl. Phys. 81, 7268 (1997).

${ }^{22}$ S. B. Samavedam and E. A. Fitzgerald, J. Appl. Phys. 81, 3108 (1997).

${ }^{23}$ J. S. Speck, M. A. Brewer, G. Beltz, A. E. Romanov, and W. Pompe, J. Appl. Phys. 80, 3808 (1996).

${ }^{24}$ B. W. Dodson and J. Y. Tsao, Phys. Rev. B 38, 12383 (1988).

${ }^{25}$ E. A. Fitzgerald, A. Y. Kim, M. T. Currie, T. A. Langdo, G. Taraschi, and M. T. Bulsara, Mater. Sci. Eng., B 67, 53 (1999).

${ }^{26}$ B. W. Dodson and J. Y. Tsao, Appl. Phys. Lett. 51, 1325 (1987).

${ }^{27}$ J. P. Hirth and J. Lothe, Theory of Dislocations (McGraw-Hill, New York, 1968).

${ }^{28}$ A. Fischer, H. Kühne, M. Eichler, F. Holländer, and H. Richter, Phys. Rev. B 54, 8761 (1996).

${ }^{29}$ R. Beresford, C. Lynch, and E. Chason, J. Cryst. Growth 251, 106 (2003). 